\title{
Conformational Redistribution of Honey Components following Different Storage Conditions
}

\author{
Giulia Cimo' and Pellegrino Conte \\ Dipartimento di Scienze Agrarie e Forestali, Università degli Studi di Palermo, Viale delle Scienze, Edificio 4, 90128 Palermo, Italy \\ Correspondence should be addressed to Giulia Cimo’; giulia.cimo@gmail.com
}

Received 8 October 2015; Accepted 1 December 2015

Academic Editor: Guillermo Moyna

Copyright ( 2015 G. Cimo' and P. Conte. This is an open access article distributed under the Creative Commons Attribution License, which permits unrestricted use, distribution, and reproduction in any medium, provided the original work is properly cited.

\begin{abstract}
The present study aims at the investigation of the changes in water distribution among the organic components of selected honey samples following honey storage at different temperatures. Results, achieved by application of fast field cycling NMR relaxometry, revealed that the organic constituents were homogeneously distributed within the whole samples stored at room temperature. Conversely, after four months of refrigeration at $4^{\circ} \mathrm{C}$, the organic systems were included in persistent clusters, as a consequence of the water release due to the larger stability of the intramolecular interactions over the intermolecular ones. The new conformational arrangements of the honey constituents entailed enhancement of honey moisture content. For this reason, it can be suggested that honey refrigeration prior to storage at room temperature may be detrimental for its long-term storage. In fact, higher risk of fermentation may occur once the sample is warmed after the first refrigeration step.
\end{abstract}

\section{Introduction}

Honey is a natural sweet and flavourful substance, produced by Apis mellifera bees [1]. It has been used since ancient times and, even nowadays, it is the only concentrated form of sugar available to mankind in most parts of the world [1].

Honey is an important product in food nutrition and mainly consists of carbohydrates (95-99\%), water, and other minor constituents (e.g., organic acids and minerals) [2]. Due to its unique composition and chemical properties, it is suitable for long-term storage, and it is easily assimilated even after long preservation.

Besides its nutritional benefits, honey appears to have also therapeutic properties [3]. In fact, it is used in dermatology for the treatment of wounds, sores, burns, ulcers, infections, and the promotion of tissue regeneration. Recently, honey has proved its powerfulness in prevention of many pathologies such as gastrointestinal disorders [4], diabetes [5], cardiovascular disease [5], and cancer [6].

Honey therapeutic properties arise from its antibacterial activity which is related to its high sugar content $[7,8]$. Most of the water molecules in honey are associated with sugars. Therefore, only few water molecules are available for microorganism activity. The latter condition generates a limiting environment for microbial growth and allows the removal of water from bacteria by osmosis, thereby further hindering bacterial life processes [9].

Bacterial activity depending on the nature and amount of water present in honey is also related to honey conservation and stability [10]. During storage, a high water content can lead to undesirable honey fermentation induced, as an example, by osmotolerant yeasts [11]. As a result, formation of ethanol and carbon dioxide occurs [12]. Ethanol can be further oxidized to acetic acid and water, thereby producing a sour taste [9].

Fermentation is the only microbiological alteration to which honey is susceptible [1] and it can result in major economic losses [13].

The key to honey long preservation is the limitation of humidity. In order to avoid fermentation risk, water content should not be above $20 \%$ of the total honey mass $[14,15]$. A proper storage temperature is also essential to reduce the risk of honey spoilage. At room storage temperatures, most types of honey take a semisolid state referred to as crystallized or granulated [1]. Crystallization results from the spontaneous precipitation of sugars to form monohydrate crystals [16], 
which may vary in number, shape, dimensions, and quality due to honey composition and storage conditions $[1,17]$. During crystallization water frees, thus increasing moisture content of the liquid phase. This condition leads to an enhancement of the fermentation risk [18].

Many authors evaluated the effect of storage conditions on honey characteristics. However, only moderate to high temperatures (above $10^{\circ} \mathrm{C}$ ) have been accounted for [19, 20]. In general, moderate temperatures (e.g., from 10 to $21^{\circ} \mathrm{C}$ ) favour crystallization. Warm temperatures (e.g., from 21 to $27^{\circ} \mathrm{C}$ ) prevent crystallization but may cause decrease in honey quality with time, due to progressive chemical and enzymatic changes [1]. These changes negatively affect honey organoleptic characteristics (such as colour, taste, and aroma) and include alterations of sugar composition (i.e., increase of disaccharides while simple sugars lower), loss of biologically active substances (e.g., inactivation of enzymatic and antimicrobial activity), and acidity increase [1]. Very warm temperatures (i.e., above $27^{\circ} \mathrm{C}$ ) prevent crystallization but favour spoilage through fermentation [19]. Only cold temperatures (around $4-5^{\circ} \mathrm{C}$ ) are considered suitable to simultaneously prevent crystallization, melting of crystallized honey, and fermentation [1].

In the present study, we aim at the investigation of physicochemical alterations occurring in honey as a consequence of two different storage conditions. Namely, honey samples were stored at room temperature and refrigerated at $4^{\circ} \mathrm{C}$ for a period of four months. After the aforementioned refrigeration interval, the samples were placed at room temperature and investigated by fast field cycling (FFC) NMR relaxometry in order to achieve information on the molecular dynamics of the complex honey mixtures.

\section{Materials and Methods}

2.1. Honey Samples. The two honey samples used in this study have been selected by beekeepers as representative of the Sicilian production from the same bee specie (i.e., Apis mellifera sicula, Dalla Torre, 1896). Their origin was assessed by palynological investigations performed by the producers. One of the honey samples was multifloral (or wildflower honey), derived from the nectar of many types of flowers. This variety was chosen as it is the most common and easy to obtain. Conversely, the second sample was a monofloral honey produced from loquat (Eriobotrya japonica (Thunb.) Lindl., 1821). The latter variety is quite rare since it is produced exclusively in a small area of Palermo (Sicily, Italy) province.

The samples were subjected to two different storage conditions: (a) storage at room temperature (RT) and (b) refrigeration (RE) at $4^{\circ} \mathrm{C}$ for a period of 4 months and then placement at room temperature till FFC NMR analyses (see below). The honey samples were stored in dark screw-cap jars. These temperatures were chosen as the most used by consumers who prefer to preserve honey either in refrigerator $\left(4^{\circ} \mathrm{C}\right)$ or at room temperature.

2.2. Fast Field Cycling $(F F C){ }^{1} \mathrm{H}$ Nuclear Magnetic Resonance (NMR) Relaxometry. The theory of fast field cycling (FFC) NMR relaxometry has been reported in other papers, such as
Conte and Alonzo [21]. For this reason, only the experimental setup used to analyse honey is reported in the present study.

${ }^{1} \mathrm{H}$ NMR dispersion (NMRD) profiles (i.e., relaxation rates $R_{1}$ or $T_{1}^{-1}$ versus proton Larmor frequencies, $\omega_{L}$ ) were acquired on a Stelar Smartracer Fast-Field-Cycling Relaxometer (Stelar s.r.l., Mede, PV, Italy) at a constant temperature $\left(25^{\circ} \mathrm{C}\right)$. The proton spins were polarized at a polarization field $\left(B_{\mathrm{POL}}\right)$ corresponding to a proton Larmor frequency $\left(\omega_{L}\right)$ of $8 \mathrm{MHz}$ for a period of polarization ( $T_{\mathrm{POL}}$ ) corresponding to about five times the $T_{1}$ estimated at this frequency. After each $B_{\mathrm{POL}}$ application, the magnetic field intensity (indicated as $B_{\mathrm{RLX}}$ ) was systematically changed in the proton Larmor frequency $\omega_{L}$ comprised in the range $0.01-10.0 \mathrm{MHz}$. The period $\tau$, during which $B_{\mathrm{RLX}}$ was applied, has been varied on 32 logarithmic spaced time sets. 32 scans were set, and the $T_{1 \max }, T_{\mathrm{POL}}$ and Recycle Delay (RD) were adjusted at every relaxation field in order to optimize the sampling of the decay/recovery curves.

Free induction decays (FIDs) were recorded following a single ${ }^{1} \mathrm{H} 90^{\circ}$ pulse applied at an acquisition field $\left(B_{\mathrm{ACQ}}\right)$ corresponding to the proton Larmor frequency of $7.20 \mathrm{MHz}$. A time domain of $100 \mu$ s sampled with 512 points was applied. Field-switching time was $3 \mathrm{~ms}$, while spectrometer dead time was $15 \mu$ s. A nonpolarized FFC sequence was applied when the relaxation magnetic fields were in the range of the proton Larmor frequencies comprised between 10.0 and 3.6 $\mathrm{MHz}$ whereas a polarized FFC sequence was applied in the proton Larmor frequencies $B_{\mathrm{RLX}}$ range of $3.0-0.01 \mathrm{MHz}$ [22].

2.3. FFC ${ }^{1} H$-NMR Data Processing. $R_{1}$ values were achieved by interpolating the ${ }^{1} \mathrm{H}$ magnetization decay/recovery curves at each $B_{\mathrm{RLX}}$ value (i.e., ${ }^{1} \mathrm{H}$ signal intensity versus $\tau$ ) with the stretched exponential function (also known as KohlrauschWilliams-Watts function) reported in (1) after exportation of the experimental data to OriginPro 7.5 SR6 (Version 7.5885, OriginLab Corporation, Northampton, MA, USA). This equation provided the best fitting with the largest coefficients of determination $\left(R^{2}>0.998\right)$. The choice of this function was due to the large sample heterogeneity resulting in a multiexponential behavior of the decay/recovery curves [23]. This approach has the advantage that it is able to handle a wide range of behaviors within a single model. For this reason, assumptions about the number of exponentials to be used in modeling NMRD data are not necessary:

$$
I(\tau)=I_{0} \exp \left[-\left(\frac{\tau}{T_{1}}\right)^{k}\right] .
$$

In (1), $I(\tau)$ is the ${ }^{1} \mathrm{H}$ signal intensity at each fixed $B_{\mathrm{RLX}}, I_{0}$ is the ${ }^{1} \mathrm{H}$ signal intensity at the thermal equilibrium, $T_{1}$ is the average proton spin lattice relaxation time, and $k$ is a heterogeneity parameter related to the stretching of the decay process. This function can be considered a superposition of exponential contributions and thus describes the likely physical picture of some distribution in $T_{1}$. The nuclear magnetic resonance dispersion (NMRD) profiles reporting the 


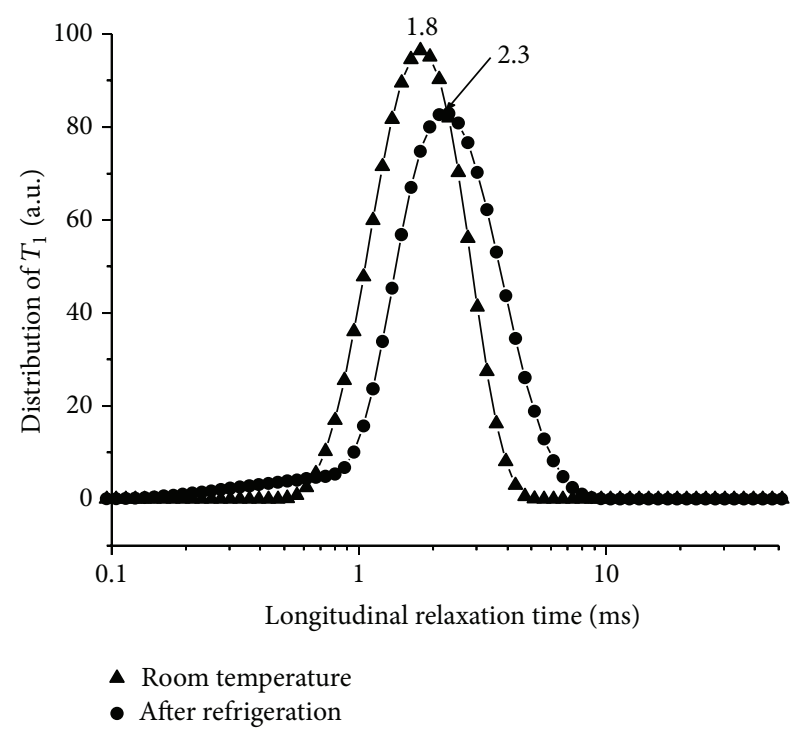

(a)

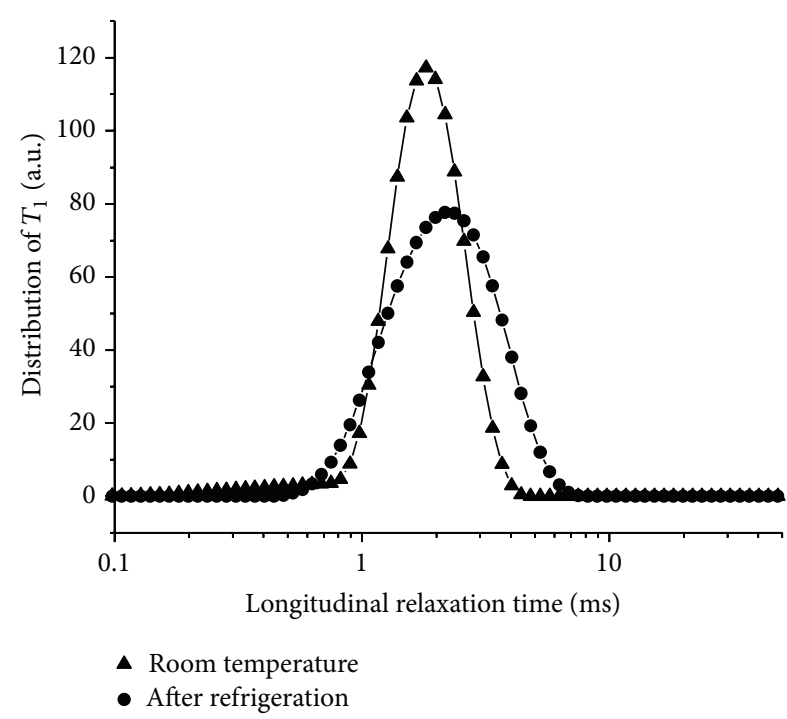

(b)

FIGURE 1: $T_{1}$ distributions of loquat (a) and multifloral (b) honey stored at room temperature (triangled dots) and after refrigeration at $4^{\circ} \mathrm{C}$ (circled dots).

calculated $R_{1}$ values $\left(T_{1}^{-1}\right)$ versus Larmor angular frequency $\left(\omega_{L}\right)$ for the honey samples are shown in Figure 2. The NMRD profiles were exported to OriginPro 7.5 SR6 and fitted with a Lorentzian function of the type [24]

$$
R_{1}=\frac{\sum_{n=1}^{N} C_{n}\left(\tau_{n} /\left(1+\left(\omega_{L} \tau_{n}\right)^{2}\right)\right)}{\sum_{n=1}^{N} C_{n}} .
$$

In (2), $R_{1}$ is the longitudinal relaxation rate and $\tau$ is the correlation time, a parameter which describes random molecular motions $[22,25]$. The number $n$ of Lorentzians that can be included in (2) without unreasonably increasing the number of parameters was determined by means of the Merit function analysis [24]. For the present study, $n=4$ was used for the mathematical fit of the NMRD profiles.

The obtained eight fitting parameters $\left(C_{1}, C_{2}, C_{3}, C_{4}, \tau_{1}\right.$, $\left.\tau_{2}, \tau_{3}, \tau_{4}\right)$ were used to retrieve an average correlation time according to the following equation [24]:

$$
\tau_{c}=\frac{\sum_{n} C_{n} \tau_{n}}{\sum_{n} C_{n}}
$$

Correlation time is a measure of the time needed for molecular reorientation and is a typical parameter for spectral density which, in turn, describes random molecular motions [22]. It represents the time necessary for a molecule to be reoriented [26]. The longer the $\tau$ value, the slower the molecular motions, thereby revealing restrictions in the motional freedom degrees of spatially restrained molecular systems. Conversely, as a molecule encompasses faster motions due to higher degrees of freedom, shorter correlation time values are expected. Results are reported in Figure 3(a).
Besides correlation times, molecular motions are characterized by activation energies $\Delta E_{a}$. Indeed, any molecular reorientation should overcome an energy barrier [26]. According to Arrhenius equation,

$$
\tau_{c}=\tau_{0} \exp \left(\frac{E_{a}}{\mathrm{RT}}\right)
$$

where $E_{a}$ is the activation energy of molecular motions and $\tau_{0}$ is the correlation time constant. Activation energies for the honey samples examined are presented in Figure 3(b).

Relaxation data at the proton Larmor frequency of $0.1 \mathrm{MHz}$ were also evaluated by UPEN algorithm (Alma Mater Studiorum, Università di Bologna, Italy) [27] to obtain the $T_{1}$ distributions at this magnetic field (Figure 1) and, therefore, information of the interactions between water and the organic component of honey.

All the determinations were made in triplicate. The fitting was done on the averaged $R_{1}$ values having a standard deviation of less than $5 \%$ of the indicated values. For this reason, differences among the NMRD dispersion profiles were judged to be significant.

\section{Results}

3.1. Effect of Storage Temperature on ${ }^{1} H N M R T_{1}$ Distributions and NMRD Profiles. Figure 1 reports the distributions of the longitudinal relaxation times $\left(T_{1}\right)$, also referred to as relaxograms, acquired at the fixed temperature of $25^{\circ} \mathrm{C}$ and at the proton Larmor frequency of $0.1 \mathrm{MHz}$, as obtained by applying the UPEN algorithm (see Section 2). Relaxation time values are associated with water mobility within the honey samples (see Section 4). In the relaxograms, $T_{1}$ values appear continuously distributed, thereby representing the different motional water populations contained in honey, 


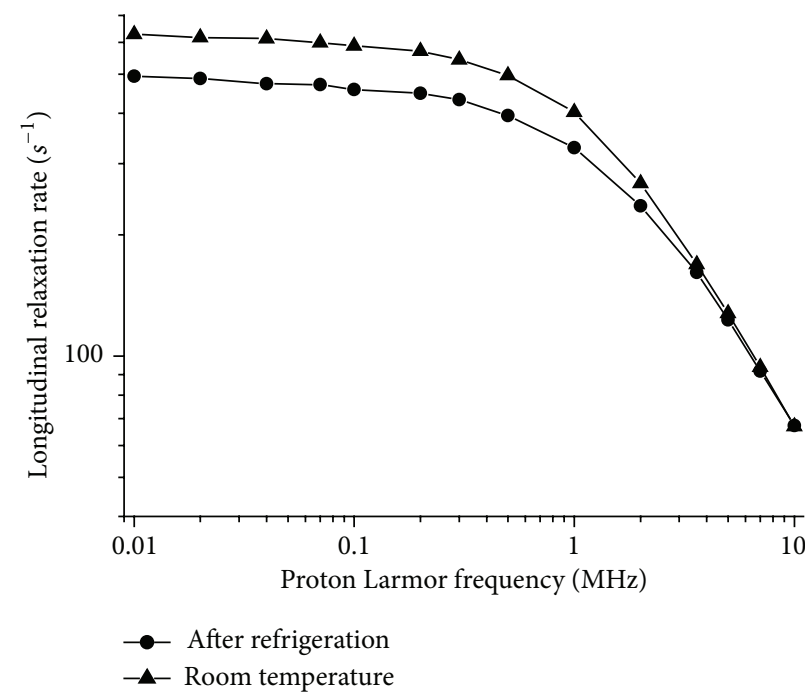

(a)

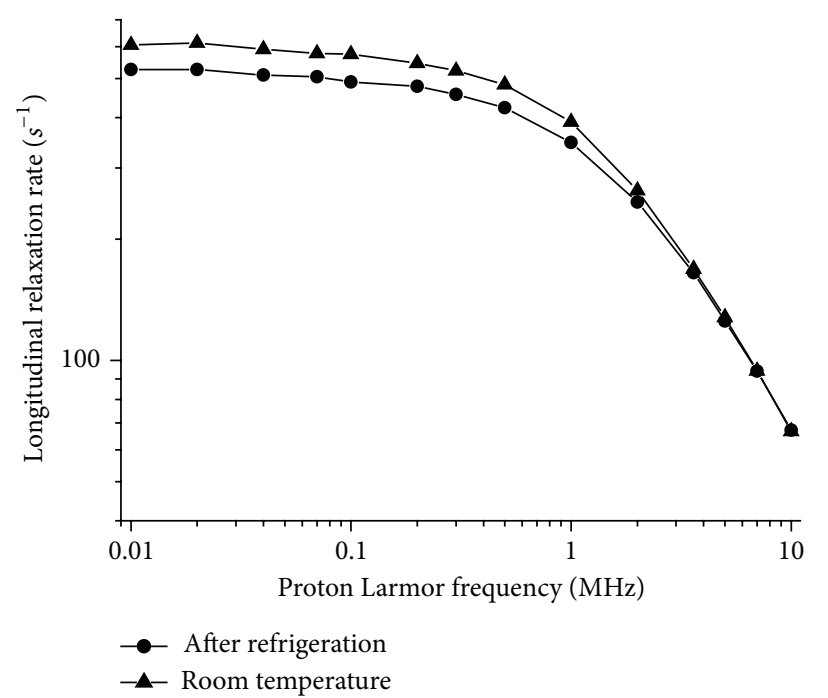

(b)

Figure 2: NMRD profiles of loquat (a) and multifloral (b) honey stored at room temperature (triangled dots) and after refrigeration at $4^{\circ} \mathrm{C}$ (circled dots).

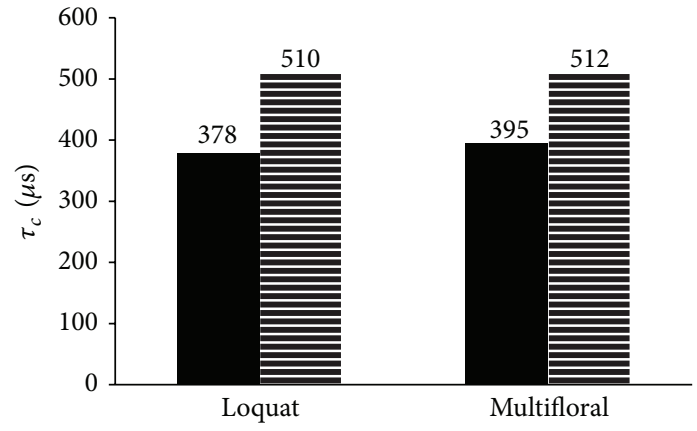

- Refrigerated = Room temperature

(a)

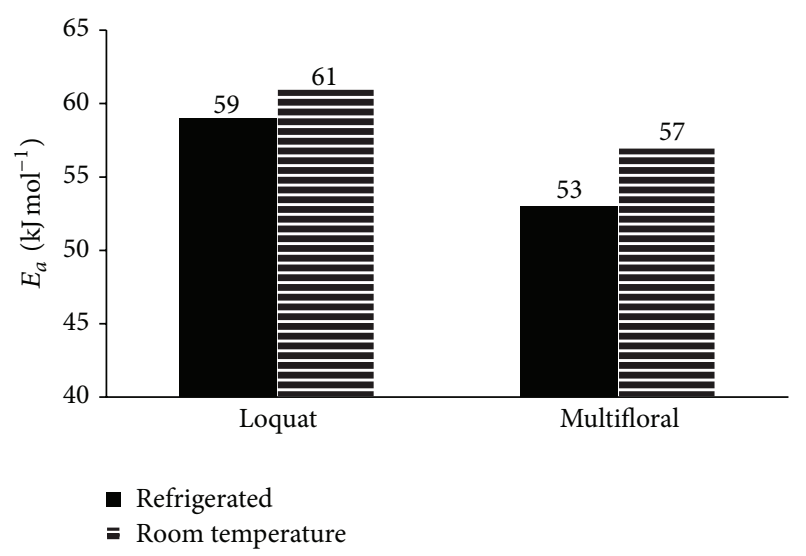

(b)

Figure 3: Correlation time, $\tau_{c}$, values (a) and activation energy, $E_{a}$, values (b) of loquat and multifloral honey stored at room temperature (striped bars) and after refrigeration at $4^{\circ} \mathrm{C}$ (black bars).

from the slow moving bound water (shorter longitudinal relaxation times) to the fast-moving bulk water (longer $T_{1}$ values).

Figure 2 shows the NMRD profiles (i.e., $R_{1}=T_{1}^{-1}$ values versus $\omega_{L}$ ) acquired at $25^{\circ} \mathrm{C}$ for loquat (Figure $2(\mathrm{a})$ ) and multifloral (Figure 2(b)) honey, stored at the conditions reported in Section 2.

The NMRD profiles of honey maintained at room temperature are placed at faster longitudinal relaxation rates as compared to the NMRD profiles of the refrigerated sample. The faster $R_{1}$ values measured for the samples kept at room temperature correspond to stronger dipolar interactions between water and honey organic components. Conversely, the slower $R_{1}$ values revealed by the refrigerated honey indicate a larger motional freedom of water molecules (see the details in Section 4).
Correlation time $\left(\tau_{c}\right)$ and activation energy $\left(E_{a}\right)$ values as retrieved by the quantitative elaboration of the NMRD profiles (see Section 2) are reported in Figure 3. Both average correlation time and activation energy values were larger for the samples kept at room temperature (striped bars) than for the cooled ones (black bars).

$\tau_{c}$ value represents the time necessary for a molecule to be reoriented [26]. The longer the $\tau_{c}$ value, the slower the molecular motions, thereby revealing restrictions in water motional freedom. Conversely, shorter $\tau_{c}$ values are related to fast molecular movements (higher degrees of freedom).

Since $\tau_{c(\mathrm{RT})}>\tau_{c(\mathrm{RE})}$ and $E_{a(\mathrm{RT})}>E_{a(\mathrm{RE})}$, the strength of the dipolar interactions between water and organic honey components can be considered stronger when honey is stored at room temperature rather than being preliminarily refrigerated. 


\section{Discussion}

4.1. Effect of Storage Temperature on ${ }^{1} H$ NMR $T_{1}$ Distributions. Distributions of longitudinal relaxation times $\left(T_{1}\right)$ at a fixed proton Larmor frequency (i.e., $0.1 \mathrm{MHz}$ in the present study) can be related to the motional behavior of protons inside multicomponent systems [21]. Proton longitudinal relaxation time values are affected by the strength of the dipolar interactions between a nucleus and its surroundings [26]. In particular, $T_{1}$ magnitude depends on the nature of the nuclei, the physical state of the system, and the temperature. The dipolar interactions between the protons participating in rigid crystalline structure are stronger and consequently produce shorter $T_{1}$ values than those in a solution state. For this reason, relaxometry is suitable for the evaluation of changes in honey structure (i.e., differentiating more ordered structures from more heterogeneous ones) when honey is subjected to different storage conditions.

In this study, the $T_{1}$ distributions (or relaxograms) of honey stored at different temperatures (Figure 1) were analysed to retrieve information on the dynamics of water molecules inside the samples. The tested honey behaves as multicomponent systems containing different motional water populations. In particular, honey is composed of water molecules which are subjected to two different dynamics. Short $T_{1}$ values (left part of the curve at around $0.3 \mathrm{~ms}$ ) can be associated with water molecules interacting intimately (or immobilized) with the organic systems (e.g., glucose and fructose) usually present in honey. In fact, hydrogen bonds between water and sugars prevent fast molecular movements, thereby enabling slow reorientation of water molecules. Conversely, longer $T_{1}$ values (right part of the curve, $>2 \mathrm{~ms}$ ) are related to water molecules which are not blocked by $\mathrm{H}$-bonds with honey organic systems but are tightly bound between them, thus forming clusters which exclude sugars. The unbound water molecules are subjected to faster molecular dynamics and provide longer $T_{1}$ values.

As shown in Figure 1, the relaxograms of honey samples first stored at low temperatures $\left(4^{\circ} \mathrm{C}\right)$ are shifted to longer $T_{1}$ values as compared to honey maintained at room temperature (peak shift from 1.8 to $2.3 \mathrm{~ms}$ ). As mentioned before, $T_{1}$ increase is representative of water mobility enhancement $[28,29]$. For this reason, we can argue that weaker interactions between organic systems and water are present in refrigerated honey as compared with the honey stored at room temperature.

The hydration of sugars contained in honey depends upon the balance between intramolecular (sugar-sugar and water-water) and intermolecular (sugar-water) hydrogen bounds [30]. The reduction of intermolecular hydrogen bonding (sugar-water) does not directly depend on the water-solute interactions, but it is a consequence of the changes in water clustering due to the storage temperature. Water molecules form an infinite hydrogen-bonded network with localized and well-structured clustering [31]. When temperature decreases, water prefers to form a less dense and more ordered (lower entropy) structure, with more extensive hydrogen bonding [32], and stronger water-water binding energies. Water cluster equilibrium shifts towards a stiffer, tetrahedrally organized structure. For this reason, small clusters of four water molecules may come together to form water bicyclo-octamers [32]. Intramolecular hydrogen bonding within water clusters increases their strength; the distances of hydrogen bonded $\mathrm{O}-\mathrm{H} \cdots \mathrm{O}$ reduce [33] and produce a reduction in water clusters volume. At $4^{\circ} \mathrm{C}$ (i.e., the temperature used in this study to refrigerate the samples), water shows its lowest volume, the size of the water hydrogenbonded cluster increases [34], and thus their persistence and stability increase [35]. However, cluster surface shows a weak hydrogen-bonding potential for adjacent molecules. It means that water cluster can make fewer and weaker hydrogen bonds with molecules that are external to the cluster (in this case with sugars). As a consequence, when temperature decreases, also sugar solubility in water drops [36], and crystallization occurs. The increase of intramolecular hydrogen bonding reduces the hydration of the carbohydrates, thereby enhancing their nonpolar character [36]. Sugars will form microaggregates with the tendency for surface minimization, excluding water. The tendency of both sugar and water to form cluster at low temperatures can be detrimental for honey storage. In fact, the decrease of intermolecular interactions with the organic components makes water more available for microorganisms, determining an increase in the risk of honey spoilage by fermentation.

4.2. Nuclear Magnetic Resonance Dispersion (NMRD) Profiles. The NMRD profiles acquired at $25^{\circ} \mathrm{C}$ for loquat and multifloral honey are reported in Figure 2. The profiles show that the longitudinal relaxation rates $\left(R_{1}\right)$ vary in the order: $R_{1(\mathrm{RT})}>$ $R_{1(\mathrm{RE})}$ in the range of proton Larmor frequencies included within 0.01 and $2 \mathrm{MHz}$.

According to the discussion above, the fastest $R_{1}$ values for the honey stored at room temperature are due to stronger ${ }^{1} \mathrm{H}-{ }^{1} \mathrm{H}$ dipolar interaction between water molecules and organic components in honey.

As mentioned before, this behavior depends on differences in water clustering resulting from different storage temperatures. At room temperature, the intermolecular hydrogen bonds between water and sugars are stronger. Protons are constrained in rigid crystalline structures which only allow slow molecular movements and reorientation. This condition produces high $R_{1}$ (and hence short $T_{1}$ ) values. Conversely, when honey is refrigerated, the intramolecular forces between water molecules prevail over the intermolecular hydrogen bonding (sugar-water), thereby determining the formation of persistent water clusters. These clusters have fewer interactions with honey carbohydrates and higher motional freedom, thereby resulting in a decrease of the protons relaxation rates.

The decrease of $R_{1}$ values with temperature increment also depends on an overall increase in water mobility. As temperature rises, hydrogen bonding strength between water and sugars molecules weakens, as well as the interactions between water molecules. The predominance of nonbonded interactions determines clustering breakdown [37] and increases water motional freedom. As a result, protons longitudinal relaxation time lengthens while relaxation rate decreases. 
The qualitative evaluation of the NMRD profiles in Figure 2 is also confirmed by their quantitative assessment through application of (1) to (3).

Figure 3(a) reports the values of the correlation times for the examined honey samples. The average correlation time $\left(\tau_{c}\right)$ is associated with the rate of molecular reorientation in the time unit [26]. Slower molecular motions, due to water movement restrictions, produce longer $\tau_{c}$ values, whereas shorter $\tau_{c}$ values are related to fast molecular movements. In particular, Figure $3(\mathrm{a})$ shows that $\tau_{c(\mathrm{RT})}>\tau_{c(\mathrm{RE})}$ in all cases, thereby confirming the aforementioned discussion.

According to the $\mathrm{H}$-bond strength among water molecules, water motion activation may require different amount of energy. The activation energy $\left(E_{a}\right)$ values of the water molecular motions were calculated by (4) and are reported in Figure $3(\mathrm{~b})$. $E_{a(\mathrm{RT})}$ resulted to be higher than $E_{a(\mathrm{RE})}$ for both loquat and multifloral honey. More energy is needed to activate water motion when honey is stored at room temperature as compared to the samples priorly subjected to refrigeration. Thus, the strength of dipolar interactions between the protons in the water system and the organic components is higher when honey is kept at room temperature with respect to refrigeration.

\section{Conclusions}

This study reports a first approach to investigate water distribution in honey samples by low field ${ }^{1} \mathrm{H} T_{1}$ NMR relaxometry. The aim was to assess the changes in the interactions between water system and organic components in honey samples stored at different temperatures. Knowledge about the nature and amount of water present in honey is important to predict honey conservation and stability. Indeed, variations in water mobility with temperature can influence microbial activity and consequently promote or reduce honey fermentation. Considering honey great value for food industry, the choice of a proper storage temperature which can prevent honey deterioration is of great importance.

The results achieved by fast field cycling NMR relaxometry revealed dramatic changes in honey component distributions, thereby suggesting conceivable enhancement of spoilage risks. Indeed, NMR investigation found substantial differences in the clustering of water and carbohydrates among honey samples kept at room temperature or chilled to $4^{\circ} \mathrm{C}$. When honey is refrigerated, the intramolecular forces between water molecules prevail over the intermolecular hydrogen bonding (sugar-water) determining the formation of persistent clusters. In turn, cluster surface shows a weak hydrogen-bonding potential for external adjacent molecules (i.e., organic sugars). As a consequence, when honey is cooled to $4^{\circ} \mathrm{C}$, sugars solubility in water decreases determining the formation of organic microaggregates which tend to minimize their external surface excluding water. This condition could be deleterious for honey preservation. Once freed, water increases its mobility and becomes more accessible to microorganisms. The latter facilitates microbial growth and could lead to an increase in the risk of fermentation. On the contrary, at room temperature, water interactions with the organic component are strong and clustering is reduced.
Since water molecules are highly associated with sugars, only few are available for bacterial activity creating a limiting environment for microbial development.

The present results are only preliminary since further studies are needed in order to confirm whether the microbial load and activity in cooled honey are greater with respect to honey kept at room temperature.

\section{Conflict of Interests}

The authors declare that there is no conflict of interests regarding the publication of this paper.

\section{Acknowledgments}

This paper has been funded by the Project APESLOW "Reintroduzione e Conservazione della Sottospecie a Rischio Estinzione Apis mellifera siciliana (Dalla Torre, 1896): Un Omaggio al Prof. Genduso" financed by Regione Sicilia (Italy). The authors are very grateful to Mr. Carlo Amodeo and Dr. Eugenia Oliveri for having provided the honey samples.

\section{References}

[1] R. Krell, Value-Added Products from Beekeeping, Food and Agriculture Organization of the United Nations, 124th edition, 1996.

[2] M. Gonnet and G. Vache, Le Miel. Composition, Propriétés et Conservation, edited by: Opida, INRA Station Expérimetale d'Apiculture, Montfavet, France, 2nd edition, 1982.

[3] V. Bansal, B. Medhi, and P. Pandhi, "Honey-a remedy rediscovered and its therapeutic utility," Kathmandu University Medical Journal, vol. 3, no. 3, pp. 305-309, 2005.

[4] S. N. Salem, "Honey regimen in gastrointestinal disorders," Bulletin of Islamic Medicine, vol. 1, pp. 358-362, 1981.

[5] N. S. Al-Waili, "Natural honey lowers plasma glucose, Creactive protein, homocysteine, and blood lipids in healthy, diabetic, and hyperlipidemic subjects: comparison with dextrose and sucrose," Journal of Medicinal Food, vol. 7, no. 1, pp. 100107, 2004.

[6] N. Oršolić, S. Terzić, L. Šver, and I. Bašić, "Honey-bee products in prevention and/or therapy of murine transplantable tumours," Journal of the Science of Food and Agriculture, vol. 85, no. 3, pp. 363-370, 2005.

[7] J. Keast-Butler, "Honey for necrotic malignant breast ulcers," The Lancet, vol. 2, no. 8198, p. 809, 1980.

[8] S. D. Somerfield, "Honey and healing," Journal of the Royal Society of Medicine, vol. 84, no. 3, p. 179, 1992.

[9] J. Chirife, M. C. Zamora, and A. Motto, "The correlation between water activity and \% moisture in honey: fundamental aspects and application to Argentine honeys," Journal of Food Engineering, vol. 72, no. 3, pp. 287-292, 2006.

[10] R. Mateo and F. Bosch-Reig, "Sugar profiles of Spanish unifloral honeys," Food Chemistry, vol. 60, no. 1, pp. 33-41, 1997.

[11] M. Jiménez, J. J. Mateo, T. Huerta, and R. Mateo, "Influence of the storage conditions on some physicochemical and mycological parameters of honey," Journal of the Science of Food and Agriculture, vol. 64, no. 1, pp. 67-74, 1994.

[12] R. D. O. R. Ribeiro, E. T. Mársico, C. D. S. Carneiro et al., "Classification of Brazilian honeys by physical and chemical 
analytical methods and low field nuclear magnetic resonance (LF ${ }^{1}$ H NMR)," LWT_Food Science and Technology, vol. 55, no. 1, pp. 90-95, 2014.

[13] J. W. White. Jr., "Composition and physical properties of honey," in Honey Review, E. Crane, Ed., pp. 157-239, Heinemann, London, UK, 1975.

[14] A. Lazaridou, C. G. Biliaderis, N. Bacandritsos, and A. G. Sabatini, "Composition, thermal and rheological behaviour of selected Greek honeys," Journal of Food Engineering, vol. 64, no. 1, pp. 9-21, 2004.

[15] G. Downey, K. Hussey, J. D. Kelly, T. F. Walshe, and P. G. Martin, "Preliminary contribution to the characterisation of artisanal honey produced on the island of Ireland by palynological and physico-chemical data," Food Chemistry, vol. 91, no. 2, pp. 347354, 2005.

[16] H. I. Assil, R. Sterling, and P. Sporns, "Crystal control in processed liquid honey," Journal of Food Science, vol. 56, no. 4, pp. 1034-1037, 1991.

[17] J. W. White Jr., "Honey," Advances in Food Research, vol. 24, pp. 287-374, 1978.

[18] L. W. Doner, "The sugars of honey-a review," Journal of the Science of Food and Agriculture, vol. 28, no. 5, pp. 443-456, 1977.

[19] L. Castro-Vázquez, M. C. Díaz-Maroto, M. A. González-Viñas, E. De La Fuente, and M. S. Pérez-Coello, "Influence of storage conditions on chemical composition and sensory properties of citrus honey," Journal of Agricultural and Food Chemistry, vol. 56, no. 6, pp. 1999-2006, 2008.

[20] M. Visquert, M. Vargas, and I. Escriche, "Effect of postharvest storage conditions on the colour and freshness parameters of raw honey," International Journal of Food Science \& Technology, vol. 49, no. 1, pp. 181-187, 2014.

[21] P. Conte and G. Alonzo, "Environmental NMR: fast-field-cycling relaxometry," in eMagRes, pp. 1-30, John Wiley \& Sons, 2014.

[22] R. Kimmich and E. Anoardo, "Field-cycling NMR relaxometry," Progress in Nuclear Magnetic Resonance Spectroscopy, vol. 44, no. 3-4, pp. 257-320, 2004.

[23] L. A. Morozova-Roche, J. A. Jones, W. Noppe, and C. M. Dobson, "Independent nucleation and heterogeneous assembly of structure during folding of equine lysozyme," Journal of Molecular Biology, vol. 289, no. 4, pp. 1055-1073, 1999.

[24] B. Halle, H. Jóhannesson, and K. Venu, "Model-free analysis of stretched relaxation dispersions," Journal of Magnetic Resonance, vol. 135, no. 1, pp. 1-13, 1998.

[25] P. Conte, V. Marsala, C. De Pasquale et al., "Nature of waterbiochar interface interactions," GCB Bioenergy, vol. 5, no. 2, pp. 116-121, 2013.

[26] V. I. Bakhmutov, Practical Nuclear Magnetic Resonance Relaxation for Chemists, John Wiley \& Sons, 2005.

[27] G. C. Borgia, R. J. S. Brown, and P. Fantazzini, "Uniform-penalty inversion of multiexponential decay data," Journal of Magnetic Resonance, vol. 132, no. 1, pp. 65-77, 1998.

[28] S. Wynne-Jones and J. M. V. Blanshard, "Hydration studies of wheat starch, amylopectin, amylose gels and bread by proton magnetic resonance," Carbohydrate Polymers, vol. 6, no. 4, pp. 289-306, 1986.

[29] F. Jaeger, A. Shchegolikhina, H. Van As, and G. E. Schaumann, "Proton NMR relaxometry as a useful tool to evaluate swelling processes in peat soils," The Open Magnetic Resonance Journal, vol. 3, no. 1, pp. 27-45, 2010.
[30] S. K. Ramadugu, Y.-H. Chung, J. Xia, and C. J. Margulis, "When sugars get wet. A comprehensive study of the behavior of water on the surface of oligosaccharides," The Journal of Physical Chemistry B, vol. 113, no. 31, pp. 11003-11015, 2009.

[31] A. Perera, R. Mazighi, and B. Kežić, "Fluctuations and microheterogeneity in aqueous mixtures," The Journal of Chemical Physics, vol. 136, no. 17, Article ID 174516, 2012.

[32] B. Bagchi, "From anomalies in neat liquid to structure, dynamics and function in the biological world," Chemical Physics Letters, vol. 529, pp. 1-9, 2012.

[33] H.-H. Limbach, P. Tolstoy, N. Pérez-Hernández, J. Guo, I. Shenderovich, and G. Denisov, "OHO hydrogen bond geometries and NMR chemical shifts: from equilibrium structures to geometric H/D isotope effects, with applications for water, protonated water, and compressed ice," Israel Journal of Chemistry, vol. 49, no. 2, pp. 199-216, 2009.

[34] W. A. P. Luck, "The importance of cooperativity for the properties of liquid water," Journal of Molecular Structure, vol. 448, no. 2-3, pp. 131-142, 1998.

[35] D. J. Anick, "Polyhedral water clusters, II: correlations of connectivity parameters with electronic energy and hydrogen bond lengths," Journal of Molecular Structure: THEOCHEM, vol. 587, no. 1-3, pp. 97-110, 2002.

[36] J. L. Dashnau, K. A. Sharp, and J. M. Vanderkooi, "Carbohydrate intramolecular hydrogen bonding cooperativity and its effect on water structure," The Journal of Physical Chemistry B, vol. 109, no. 50, pp. 24152-24159, 2005.

[37] O. Mishima and H. E. Stanley, "The relationship between liquid, supercooled and glassy water," Nature, vol. 396, no. 6709, pp. 329-335, 1998. 

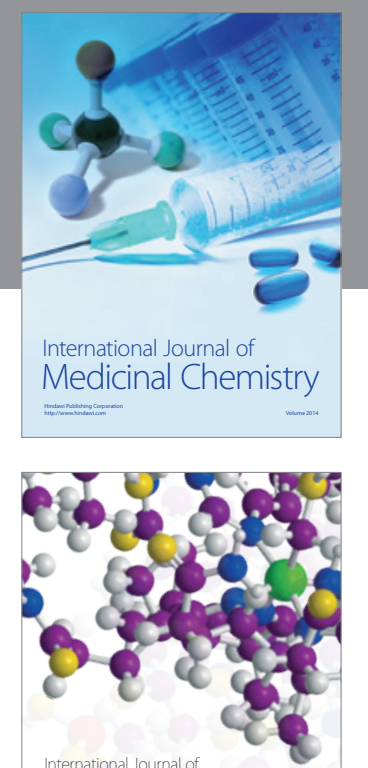

\section{Carbohydrate} Chemistry

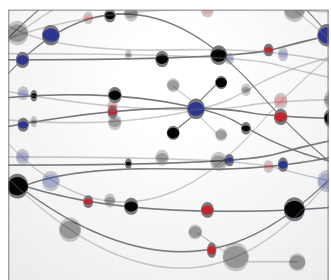

The Scientific World Journal
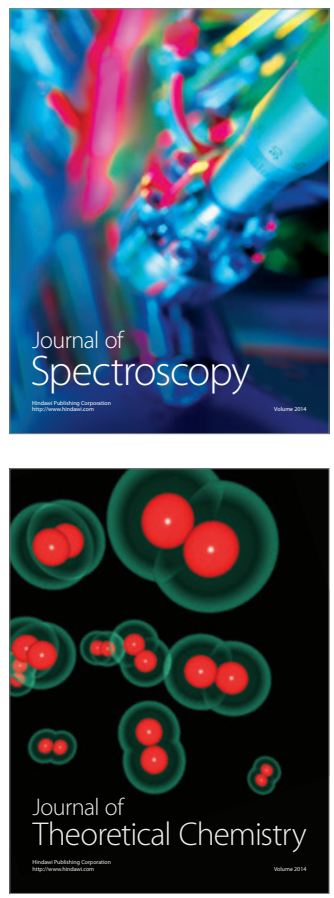
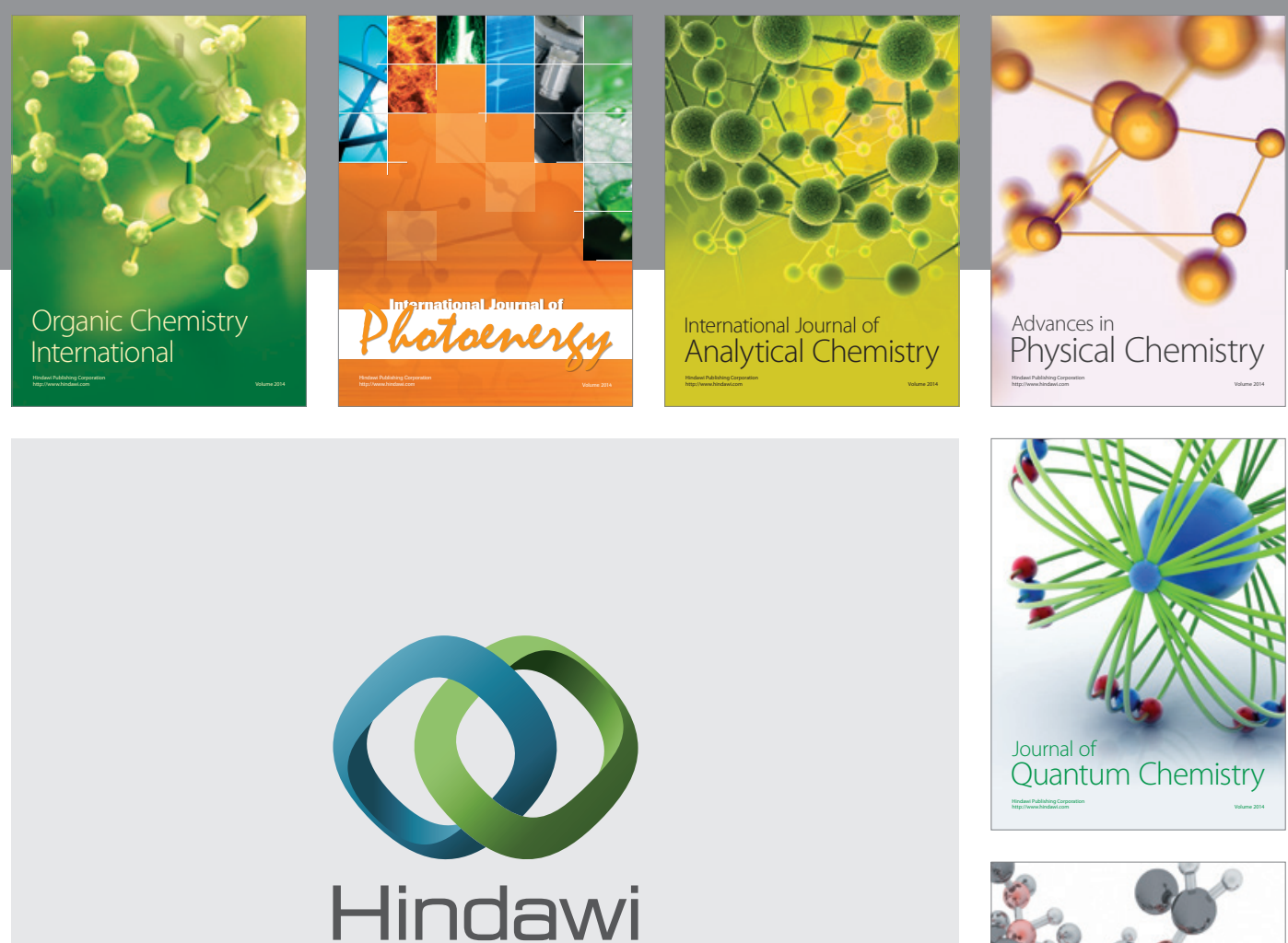

Submit your manuscripts at

http://www.hindawi.com

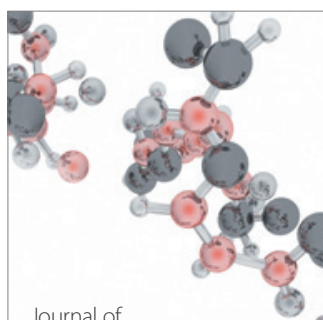

Analytical Methods

in Chemistry

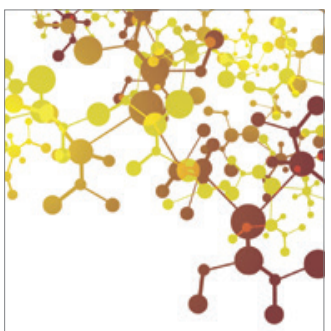

Journal of

Applied Chemistry

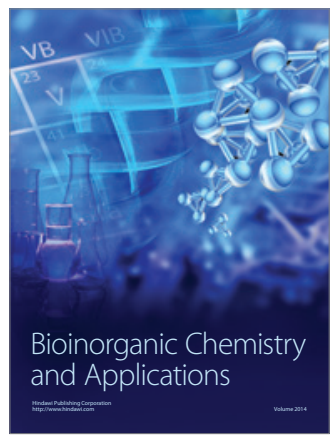

Inorganic Chemistry
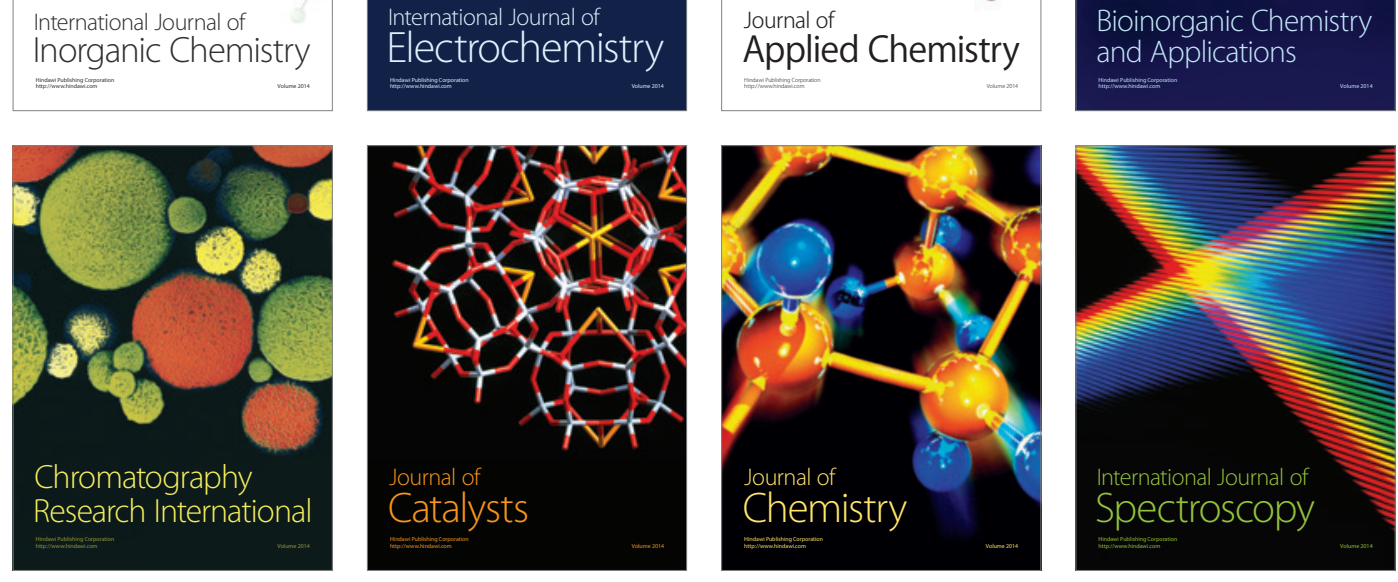